\title{
L'HÉRITABILITÉ DE LA PONTE D'HIVER DE POULETTES RECEVANT OU NON UN ÉCLAIREMENT SUPPLEMENTAIRE
}

\author{
PAR \\ P. MERAT \\ Avec la rollaboration technique de Odile ARGAxD. \\ Station de Recherches Avicoles, C. N. R. Z., Jouy-en-Josas (S.-et-O.).
}

\section{SOMMAIRE}

Pendant 4 années de suite, les poulettes des familles de frères et scurs d'un troupeau de sélection ont été réparties au hasard, par moitié, à l'âge de Io semaines, dans deux poulaillers : l'un soumis au jour naturel, l'autre avec en plus un éclairage additionnel procurant aux pondeuses 44 heures de "jour ".

Les poulettes étaient nées en mars-avril et au nombre d'environ I 200 au total dans chacun des deux groupes.

I'héritabilité de l'âge au premier ouf et du nombre d'cufs pondus respectivement jusque fin octobre, fin novembre et fin décembre, était comparée pour les poulettes éclairées ou non.

Les résultats font apparaître une héritabilité plus grande, dans l'ensemble, en poulaillers "éclairés "; ainsi, l'héritabilité moyenne de la ponte $\left(\frac{h^{2} d+h^{2} s}{2}\right)$ jusque fin décembre est de 0,53 contre 0,3 I en poulaillers non éclairés; les valeurs correspondantes pour l'âge au premier cuf sont 0,44 et 0,17 . Cela suggère la possibilité d'un progrès plus rapide par sélection pour la ponte sur un troupeau éclairé.

\section{IN'TRODUC'TION}

I'éclairage des pondeuses est un moyen simple d'augmenter la production d'automne et d'hiver. Cette pratique est largement répandue chez les producteurs d'œufs de consommation.

On n'a pas étudié en détail les répercussions possibles de cette technique sur les méthodes de sélection ou d'accouplement des reproducteurs. 
D'une façon plus générale, peu d'études ont visé à déceler des différences de manifestation du génotype pour la ponte suivant le régime d'éclairement donné aux volailles, ou à faire apparaître des différences en modifiant ce régime. On possède surtout des observations éparses faites à l'occasion d'autres recherches.

\section{A. - Variation du facteur lumière et différences génétiques pour la ponte}

Les principales observations ont porté sur les différences génétiques manifestées lorsqu'on augmente artificiellement la durée d'éclairement en hiver. Nous les avons résumées dans le tablean suivant:

\begin{tabular}{|c|c|c|c|}
\hline Auteurs & Animaux étudiés & $\begin{array}{c}\text { Effet } \\
\text { de l'éclairage additionnel } \\
\text { par rapport } \\
\text { au lot témoin }\end{array}$ & $\begin{array}{l}\text { Critère } \\
\left.\text { étudié ( }{ }^{1}\right) \\
\text { (sur les } Q \text { ) }\end{array}$ \\
\hline $\begin{array}{l}\text { SCOTT et PAYNe, I937. } \\
\text { HOFFMANN et HANCOCK, } \\
\text { I950. }\end{array}$ & $\begin{array}{l}\text { Pintades. } \\
\text { Pintades. } \\
\text { Oies. }\end{array}$ & $\begin{array}{l}\text { Pintade : pas d'effet (diffé- } \\
\text { rence avec les autres es- } \\
\text { pèces. Oies de } 6 \text { ans : } \\
\text { ponte stimulée.) }\end{array}$ & MS \\
\hline WILCKE, I939. & $\begin{array}{l}\text { Jeunes dindes : } \\
\text { Bronzées. } \\
\text { White Iolland (éclairage } \\
\text { à partir du I-I). }\end{array}$ & $\begin{array}{l}\text { White Holland rendues } \\
\text { plus précoces de } 15 \text { jours } \\
\text { à } 3 \text { semaines. }\end{array}$ & MS \\
\hline Davis et Phinquite, 1942. & $\begin{array}{l}\text { Pintades: } \\
\text { varićté "White "; } \\
- \text { "Pearl " } \\
\text { éclairage continu à partir } \\
\text { du } 3 I-I \text { ). }\end{array}$ & $\begin{array}{l}\text { "White " rendues plus pré- } \\
\text { coces de } 4 \text { semaines, mais } \\
50 \% \text { de ponte obtenu } \\
\text { en même temps sur tous } \\
\text { les groupes. }\end{array}$ & MS \\
\hline $\begin{array}{l}\text { Nicholas, Callenbach, } \\
\text { Mlrphy, I } 944 .\end{array}$ & $\begin{array}{l}\text { Poules : } \\
\text { Plymouth Rock; } \\
\text { Leghorn. } \\
\text { (éclairage le matin). }\end{array}$ & $\begin{array}{l}\text { Réponse des Plymouth } \\
\text { Rock à l'augmentation } \\
\text { d'éclairement moins im- } \\
\text { portante que celle des } \\
\text { Leghorn. }\end{array}$ & $\mathrm{PH}$ \\
\hline $\begin{array}{l}\text { AsMunioson, LoREntz et } \\
\text { Moses, i } 946 \text {. }\end{array}$ & $\begin{array}{l}\text { Dindes: } \\
\text { (plusieurs lignées). }\end{array}$ & $\begin{array}{l}\text { Deux fois de suite : matu- } \\
\text { rité sexuelle hátée sur } \\
\text { une lignée " précoce ", } \\
\text { proportionnellement à } \\
\text { l'intensité lumineuse, em- } \\
\text { ployée ; pas d'effet sur } \\
\text { une lignée "tardive ". }\end{array}$ & MS \\
\hline KARAPETJAN, I953. & $\begin{array}{l}\text { Poulettes : } \\
\text { race locale; } \\
\text { Leghorn. }\end{array}$ & $\begin{array}{l}\text { Réponse jlus importante } \\
\text { pour la race locale. }\end{array}$ & MS \\
\hline KARAPETJAN, I954. & Poules : & " & IP \\
\hline $\begin{array}{l}\text { OsBoRNe, I954. } \\
\text { (Apparemment, première } \\
\text { étude génétique quanti- } \\
\text { tative dans ce domaine). }\end{array}$ & $\begin{array}{c}\text { Poulettes Leghorn dorées } \\
\text { de plusieurs familles. }\end{array}$ & $\begin{array}{l}\text { Les différences d'origine gé- } \\
\text { notypique voient leur } \\
\text { importance relative a- } \\
\text { moindrie dans la varia- } \\
\text { bilité totale. }\end{array}$ & MS \\
\hline
\end{tabular}

(1) MS = Maturité sexuelle ; $\mathrm{PH}=$ Ponte d'hiver ; IP $=$ Intensité de ponte.

On peut ajouter à ces observations celles portant sur des différences individuelles de réponse à la stimulation par un ćclairage additionnel (BYFrl,y, I948, sur la poule ; BissonNetTe et CSECH, I938, Harír, 
I949, Asmundson et Moses, I950, sur diverses espèces) ou sur les différences individuelles de ponte existant dans l'obscurité totale (WILsoN et WOODARD, I958, sur la poule).

\section{B. - Corrélation entre les performances obtenues avec divers traitements lumineux}

Plusieurs auteurs se sont inquiétés de la relation possible entre la ponte dans les conditions naturelles et la "sensibilité " aux variations du rythme diurne d'éclairement.

Pour Whetham, I933, les pondeuses médiocres sont aussi les plus affectées par la variation de durée du jour; on peut se demander si cette relation ne découle pas, au moins en partie, de la définition même des " bonnes» ou " mauvaises » pondeuses. Il s'agit ici des saisons et non spécifiquement du facteur "lumière ".

RoMANOFF, I949, et HutT, I949, émettent une opinion analogue.

Selon BissonnetTe et CsEch, I938, les poules faisanes pondant le plus avec une lumière additionnelle sont aussi moins affectées par une diminution d'éclairement.

WiLson et ABPLANALP, r956, font une observation analogue sur des poules: Les meilleures pondeuses sont plus résistantes aux chocs produits par des changements de rythme d'éclairement en chambre conditionnée.

Dans ces corrélations observées, on ne sait quelle part revient au génotype ; une telle analyse resterait à faire.

\section{C. - Remarques d'ensemble et objet du présent travail}

Nous pouvons tirer de ces résultats quelques remarques d'ensemble. I $^{\circ}$ On ne sait dans quelle mesure les différences génotypiques observées doivent être rapportées aux conditions expérimentales, ou à la variation d'éclairement qu'apporte le traitement par rapport à la période préexpérimentale : On connaît l'effet de cette variation sur le cycle sexuel, et les observations citées n'ont duré que quelques mois (sauf KARAPETJAN, I954).

$2^{\circ}$ Certains estiment qu'un éclairage additionnel, nivelant par le haut les performances, doit réduire la variance d'origine génétique pour la ponte (Whetham, I933; HutT, I949).

Au contraire, DobIE, Carver et RobErTs, I946, écrivent que les conclusions de WHETHAM " ne sont pas en accord avec les pratiques actuelles de sélection ". De même, Bowman et ARCHIBALD, I959, pensent qu'une diminution $d u$ cofficient de variation (produite par la stimulation 
lumineuse de la ponte) devrait augmenter l'héritabilité du nombre d'œufs pondus.

Il s'agit dans tous les cas d'hypothèses, qui seraient à vérifier expérimentalement.

Le premier groupe d'opinions paraît confirmé par le travail d'OsBORNE, I954, où la variabilité génotypique pour l'âge au premier œuf est fortement réduite par l'éclairage artificiel.

Mais d'autres observations citées montrent que des différences génétiques sont manifestées grâce à la lumière artificielle, pour la maturité sexuelle chez diverses espèces (WILcke, Daris et Penquite; AsMundSON, I IORENTZ et MOSES).

$3^{\circ}$ Tous ces exemples suggèrent une manifestation différente du génotype en présence d'éclairements différents. Ils indiquent en même temps l'existence de différences génétiques dans la " sensibilité " à certains stimuli lumineux.

$4^{\circ}$ On peut en rapprocher les observations sur les interactions statistiques entre le génotype des poules pour la maturité sexuelle et leur

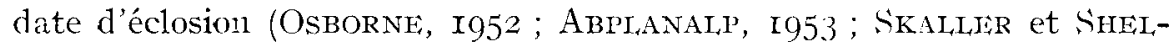
DON, I954) ou sur l'héritabilité différente de l'âge au prenier œuf suivant la date d'éclosion (MÉRAT, 1956). Ces observatio'ns suggèrent, chez la poule, l'existence de gènes de "sensibilité " aux conditions défavorables causées ou rendues opérantes par la dininution de durée du jour à l'automne.

Au total, le problème a été très peu analysé par les méthodes de la génétique quantitative : Bitude statistique des interactions génotypex éclairement, introduction de la notion de corrélation génétique entre les performances dans deux milieux différeits (FALCONIR, I952) et comparaison de l'héritabilité des performances dans des conditions d'éclairement différentes.

Ce dernier point est l'objet du présent travail : I’héritabilité des caractères de ponte diffère-t-elle quand on l'estime sur un troupean de pondeuses recevant un éclairement supplémentaire, ou sur un troupeau recevant le jour naturel?

\section{MATÉRIEL, ĖT MÉTHODES}

\section{Animaux.}

Le troupean étudié était issu au départ (I954) d'un croisement Rhode Island $\times$ Gâtinaise, et sélectionné depuis, d'une part pour un ensemble d'aptitudes économiques, d'autre part pour l'homogénéité des caractères extérieurs (MÉRAT, I955). 


\section{Locaux et conditions d'élevage.}

Sur quatre années (I956, I957, I958 et I959) les poulettes, nées en mars-avril, ont été réparties à ı semaines dans deux poulaillers de ponte de chacun 4 à 500 pondeuses, de construction identique, et oì l'élevage était conduit de façon semblable. Pour l'un d'eux (poulailler "éclairé "), un interrupteur horaire donnait un supplément de lumière le matin et le soir, depuis le 20 août, fournissant aux animaux une durée totale d'éclairement journalier de I4 heures (jour naturel + lumière artificielle). L'autre poulailler, que nous désignerons par la mention "non éclairé ", ne recevait que le jour naturel. L'éclairage additionnel était fourni par des lampes de 75 watts espacées de $5 \mathrm{~m}$ environ, ce qui correspond à la pratique usuelle, de même que la durée de $\mathrm{I}_{4}$ heures, considérée en général comme un optimum.

L.es éclosions (pedigree) s'étalaient sur 4 semaines en $\mathrm{x} 95^{6}$, 6 en I957, I958 et I959. Les poulettes écloses le même jour étaient, à Io semaines, réparties en nombre égal dans les deux poulaillers, les numéros pairs dans l'un, impairs dans l'autre. Chaque famille était ainsi répartie à peu près par moitié dans les deux locaux, et en principe "au hasard " en ce qui concerne le génotype des animaux.

I,es mêmes poulaillers ont été utilisés pendant les quatre années, mais le poulailler éclairé a été alterné d'une année sur l'autre, pour éviter autant que possible de superposer un effet " poulailler " à. l'effet " éclairage"

L'entrée en ponte s'échelonnait de fin juillet à octobre.

\section{Critères étudiés.}

I es caractères étudiés, sur toutes les années, sont l'âge au premier œuf, et le nombre cumulé d'œufs pondus jusque respectivement fin octobre, fin novembre et fin décembre.

Les moyennes et variances pour ces différents caractères sont données dans les tableaux I à IV, ainsi que les composantes " père " et "mère " de la variance, estimées conformément au modèle décrit par KING et Henderson, I954, adapté au cas de classes inégales, et permettant l'estimation de l'héritabilité.

Cette estimation correspond donc à celle des corrélations intraclasse entre sœurs et demi-sœurs de même père, la classification étant hiérarchique.

Pour I957, I958 et I959, où les dates d'éclosion sont échelonnées sur 6 semaines, premières et dernières couvées sont séparées et le calcul des composantes de la variance est corrigé pour l'effet de la date d'éclo- 
sion (cf. KING et HENDEkson, I954). Cet effet est également retranché dans le calcul des variances données ci-dessous (tableau I).

\section{TABLEAU I}

Nombre d'animaux, moyennes et variances.

\begin{tabular}{|c|c|c|c|c|c|c|c|c|c|c|c|c|}
\hline & \multirow{2}{*}{\multicolumn{3}{|c|}{$\begin{array}{c}\text { Age } \\
\text { au premier ouf } \\
\text { (en jours) }\end{array}$}} & \multicolumn{9}{|c|}{ Nombre d'œufs pondus jusque: } \\
\hline & & & & \multicolumn{3}{|c|}{ fin octobre } & \multicolumn{3}{|c|}{ fin novembre } & \multicolumn{3}{|c|}{ fin décembre } \\
\hline & $\mathrm{N}$ & $\bar{x}$ & $s^{2}$ & $\mathrm{~N}$ & $\bar{x}$ & $s^{2}$ & $\mathrm{~N}$ & $\bar{x}$ & $s^{2}$ & $\mathrm{~N}$ & $\vec{x}$ & $s^{2}$ \\
\hline $\begin{array}{l}1956 \text { : } \\
\text { Poulettes écl } \\
\text { Poulettes no }\end{array}$ & $3 \circ 3$ & I 75,3 & 667 & 259 & 31,6 & 244 & 259 & $4^{6,3}$ & 359 & 259 & 54,0 & $50 \mathrm{I}$ \\
\hline $\begin{array}{l}\text { rées ......... } \\
\quad \text { r957: }\end{array}$ & 29.3 & 184,0 & 966 & 246 & 27,1 & 258 & 246 & 40,8 & $46 I$ & 246 & 45,6 & 559 \\
\hline $\begin{array}{l}\text { Poulettes éclairées. } \\
\text { Poulettes non éclai- }\end{array}$ & 359 & I 80,7 & 64.3 & 317 & 24,9 & 222 & 357 & $3^{6,8}$ & 372 & $3+7$ & 49,0 & 569 \\
\hline $\begin{array}{l}\text { rées } \ldots \ldots \cdots \cdots \\
\quad 195^{8}:\end{array}$ & 362 & 182,2 & I I 34 & 329 & $2 \mathrm{I}, 4$ & $26 \mathrm{I}$ & 329 & 28,9 & 420 & 329 & $3^{6,0}$ & $62 \mathrm{I}$ \\
\hline $\begin{array}{l}\text { Poulettes éclairées. } \\
\text { Poulettes non éclai- }\end{array}$ & 395 & $\mathrm{I} 8 \mathrm{I}, 5$ & $52 \mathrm{I}$ & $35^{8}$ & 27,9 & 223 & $35^{8}$ & $41, \mathrm{I}$ & 335 & $35^{8}$ & $5^{2, \mathrm{x}}$ & 521 \\
\hline $\begin{array}{c}\text { rées } \ldots \ldots \ldots \ldots \\
\quad 1959:\end{array}$ & 400 & 183,5 & $80 \mathrm{I}$ & $3^{6} 5$ & 24,3 & 217 & $3^{6} 5$ & 30,7 & 354 & $3^{65}$ & 34,6 & 469 \\
\hline $\begin{array}{l}\text { Poulettes éclairées. } \\
\text { Poulettes non éclai- }\end{array}$ & $19 \mathrm{I}$ & I 69,3 & 646 & 187 & $3 I, 3$ & 286 & 185 & 43,3 & $3^{87}$ & 182 & 57,9 & $47^{2}$ \\
\hline rées $\ldots \ldots \ldots$ & 184 & I 77,3 & 1322 & 177 & 25,7 & 260 & 175 & 33,4 & 375 & 174 & $4,3,4$ & 507 \\
\hline $\begin{array}{l}\text { Total : } \\
\text { Poulettes éclairées. } \\
\text { Poulettes non éclai- } \\
\text { rées } \ldots \ldots \ldots \ldots \ldots\end{array}$ & $\begin{array}{ll}\text { I } 248 \\
\text { I } 239\end{array}$ & $\begin{array}{l}177,9 \\
182,3\end{array}$ & $\begin{array}{r}6 \mathrm{ro} \\
\mathrm{I} \text { OI } 4\end{array}$ & $\begin{array}{l}\text { I } \begin{array}{l}\text { I } 2 \\
\text { I I }\end{array} \\
\text { I I I I } 7\end{array}$ & $\begin{array}{l}28,5 \\
24,3\end{array}$ & $\begin{array}{r}238 \\
246\end{array}$ & 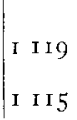 & $\begin{array}{l}41,5 \\
32,8\end{array}$ & $\begin{array}{l}359 \\
400\end{array}$ & $\begin{array}{lll}\text { I } & \text { I I } 6 \\
\text { I } & \text { I I } 4\end{array}$ & $\begin{array}{l}52,6 \\
38,8\end{array}$ & $\begin{array}{l}522 \\
540\end{array}$ \\
\hline
\end{tabular}

I.es distributions de fréquences ne sont pas exactement normales (en particulier, dissymétrie des distributions d'âges au premier œuf), mais à un degré qui n'a pas parı assez important pour rendre nécessaire une transformation des données.

\section{RÉSULTATS}

IO LES MOYENNES E'T VARIANCES pour les poules éclairées ou non pour les diverses années et les 4 caractères étudiés (1) sont données dans le tableau I (pour le total, les variances données sont intra-années),

I a différence d'âge moyen au premier œuf est de l'ordre de quelques jours seulement; quand l'effet de l'éclairement additionnel commençait à se faire sentir, une bonne proportion des poulettes étaient déjà entrées en ponte.

(1) Ces caractères portent sur les survizantes jusqu'au delà du 3i décembre pour le nombre d'œufs; de même, pour l'âge au premier cuf, les poules non entrées en ponte au 3I décembre n'ont été comptées que si elles ont survécu au-delà du 3 r décembre, sauf en 1959 , où la mortalité était la plus faible. 
Pour le nombre d'œufs pondus, les différences sont nettes en faveur du lot éclairé, comme on pouvait s'y attendre (1).

On remarque de plus la variance très significativement moindre de l'âge au premier œuf chez les poules éclairées, le nombre de (retardataires »

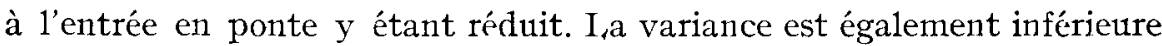
pour les nombres d'œufs pondus, mais la différence est moins importante.

I,es coefficients de variation (tableau II) présentent une différence plus accusée, du fait des moyennes supérieures en poulaillers éclairés.

TABLEAU II

Coefficients de variation.

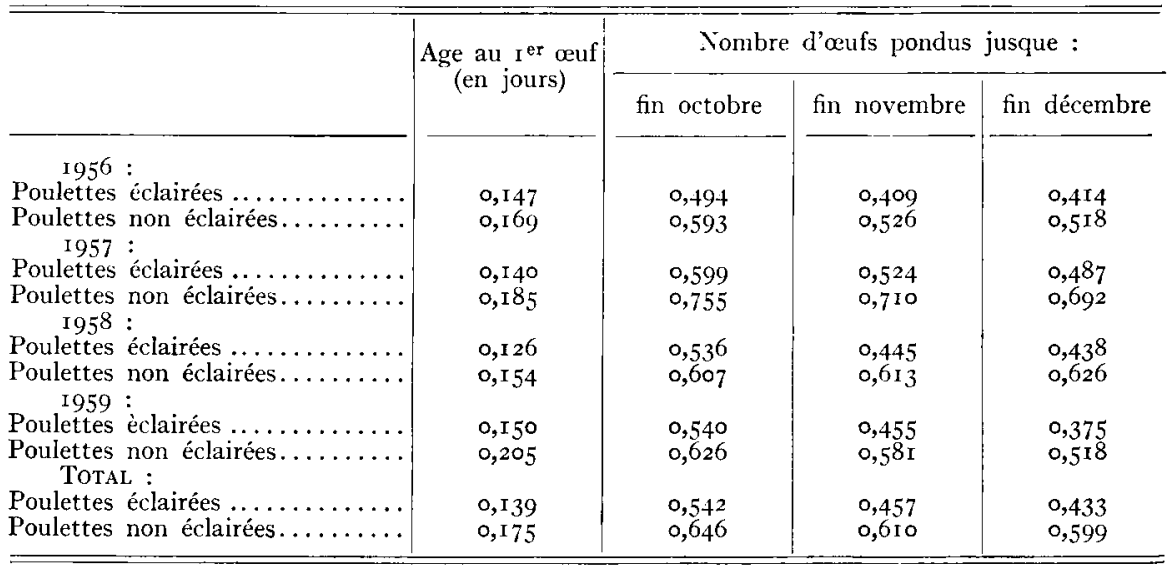

L'éclairement, en fin de compte, a surtout pour effet de réduire la proportion des pondeuses les plus mauvaises ou les plus " retardées", ce que vérifie l'examen des distributions de fréquences. Ce fait a déjà été signalé (HuTt, I949).

\section{$2^{\circ}$ LES HÉRITABILITÉS.}

Les composantes de la variance pour les divers caractères sont données par année dans le tableau qui suit (tableau III).

Nous appelons

$\mathrm{N}$ le nombre d'enfants.

$d$ le nombre de mères.

$s$ le nombre de pères.

$\mathrm{s}_{e}{ }^{2}$ la composante "résiduelle " de la variance, dûe aux écarts entre sœurs (contenant la plus grande partie de la variance "milieu", plus une part de la variance génotypique).

( $\left.{ }^{1}\right)$ La ponte n'était pas relevée au nid-trappe les dimanches et jours fériés. De toutes façons, les conditions d'élevage n'étaient pas celles d'une production intensive, ce qui se reflète dans les pourcentages de ponte. 


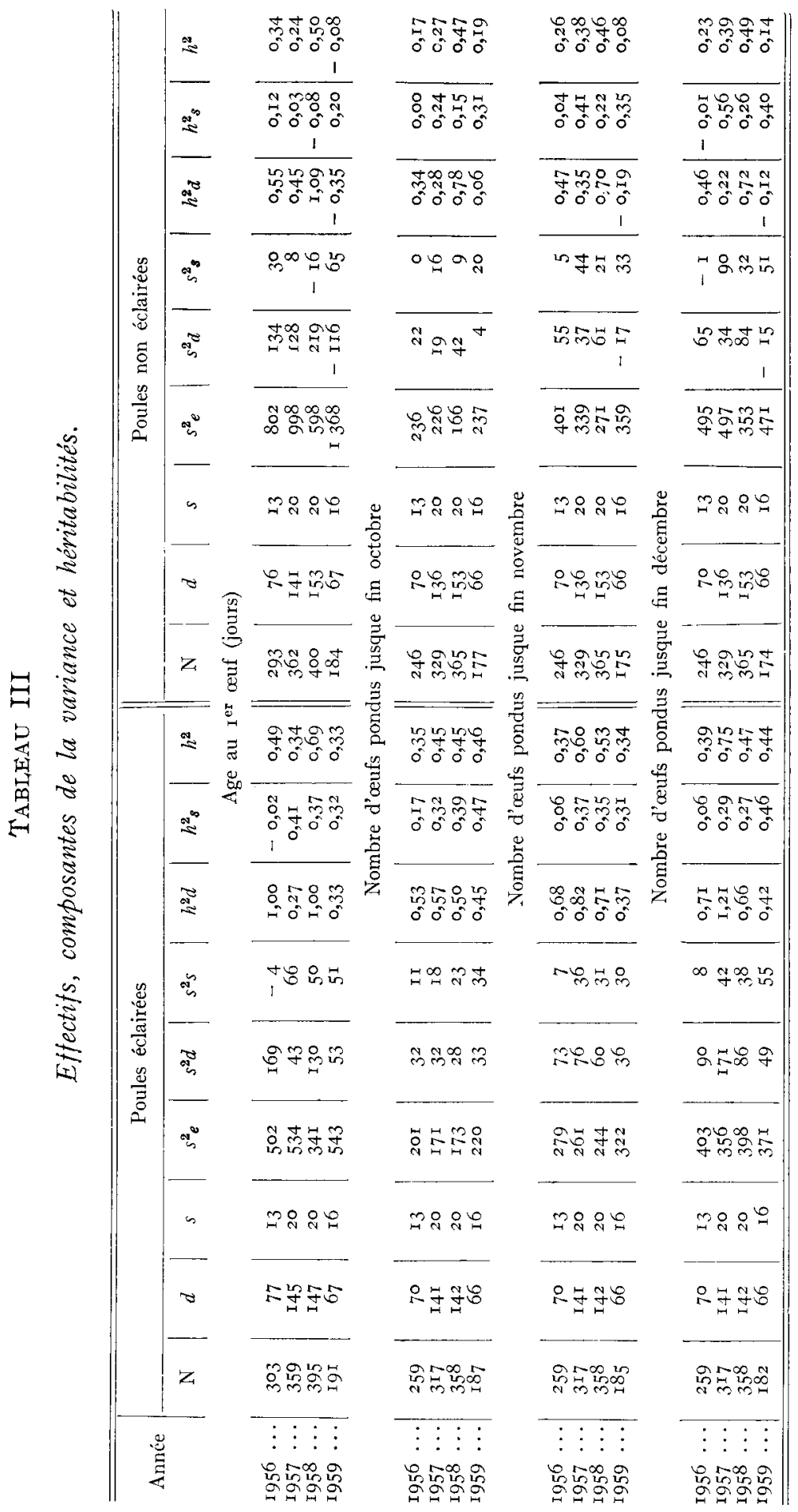


$\mathrm{s}_{d^{2}}$ la composante "mère " de la variance, dûe à l'apport commun de la mère à tous ses enfants.

$s_{s}{ }^{2}$ la composante " père " de la variance, dûe à l'apport commun du père à tous ses enfants.

On sait (LERNER, I950, p. I20) que, si l'accouplement des reproducteurs a lieu "au hasard " et s'il n'y a pas de corrections dûes à la consanguinité, on peut calculer deux estimations de l'héritabilité :

$$
h^{2}{ }_{d}=\frac{4 s^{2}{ }_{a}}{s^{2}+s^{2}{ }_{d}+s^{2}} \quad \text { et } \quad h^{2}{ }_{s}=\frac{4 s^{2} s}{s^{2}{ }_{e}+s^{2}{ }_{d}+s^{2}{ }_{s}}
$$

ainsi que leur moyenne

$$
h^{2}=\frac{2\left(s^{2} d+s^{2} s\right)}{s_{e}^{2}+s^{2} d+s^{2}} .
$$

Pour simplifier, nous n'avons pas mentionné daus le tableau III les sommes de carrés et degrés de liberté ayant servi à calculer les composantes de la variance.

I'estimation des héritabilités pour les données groupées sur une base intra-années est donnée dans le tableau IV.

TABLEAC IV

\begin{tabular}{|c|c|c|c|c|c|c|}
\hline & \multicolumn{3}{|c|}{$\begin{array}{l}\text { Composantes } \\
\text { de la variance }\end{array}$} & \multicolumn{3}{|c|}{ Héritabilités } \\
\hline & $s_{\rho}^{2}$ & $s_{d}^{2}$ & $s_{s}^{2}$ & $h_{d}^{2}$ & $h^{2}$ & $h^{2}$ \\
\hline \multicolumn{7}{|l|}{ Age au I er œuf : } \\
\hline Poulettes éclairées & 475 & 94 & 38 & 0,62 & 0,25 & 0,44 \\
\hline Poulettes non éclairées...$\ldots \ldots$ & 923 & $7 \mathrm{I}$ & 15 & 0,28 & 0,06 & 0,17 \\
\hline Eufs pondus jusque fin octobre : & & & & & & \\
\hline Poulettes éclairées..$\ldots \ldots \ldots \ldots \ldots \ldots$ & I92 & 25 & 19 & 0,42 & 0,32 & 0,37 \\
\hline $\begin{array}{l}\text { Poulettes non éclairées } \ldots \ldots \ldots \ldots \ldots \ldots \\
\text {. }\end{array}$ & 215 & 20 & IO & 0,32 & 0,16 & 0,24 \\
\hline \multicolumn{7}{|l|}{ Eufs pondus jusque fin novembre : } \\
\hline Poulettes éclairées $\ldots \ldots \ldots \ldots \ldots$ & 287 & 48 & 27 & 0,53 & 0,29 & $0,4 \mathrm{I}$ \\
\hline l'oulettes non éclairées .......... & 340 & 33 & 23 & 0,33 & 0,24 & 0,29 \\
\hline $\begin{array}{l}\text { s pondus jusque fin décembre : } \\
\text { Poulettes éclairées ............... }\end{array}$ & 379 & IO4 & $3 \mathbf{I}$ & $0,8 \mathrm{I}$ & 0,24 & $0,5,3$ \\
\hline Poulettes non éclairées ............ & $45^{2}$ & 43 & $4 \mathrm{I}$ & 0,32 & 0,30 & 0,31 \\
\hline
\end{tabular}

Données groupées.

\section{INTERPRÉTATION DES RÉSULTATS}

I. - Le principal fait qui se dégage est l'héritabilité plus gra1nde, dans l'ensemble, chez les poules éclairées, que ce soit pour l'âge au premier ouf ou pour la ponte jusqu'aux diverses dates. Cela se produit les quatre années, sauf pour la ponte jusque fin octobre et fin décembre I958, où l'héritabilité est à peu près égale dans les deux groupes.

Les erreurs d'échantillonnage sont importantes. On ne peut appli- 
quer, en toute rigueur, le test d'Osborne et PATERSON, I952, puisque les effectifs sont variables dans les sous-classes. Nous avons cepeudant, à titre indicatif, applipué ce test à une population donnant les mêmes résultats que la nôtre. ayant les mêmes nombres d'animaux et de reproducteurs, mais dont les effectifs dans les sous-classes seraient égaux : Dans le cas des héritabilités de la ponte fin décembre obtenues par la composante " mère " de la variance, la différence entre lots éclairés on $110 n$ serait à peu près à la limite du seuil 5 p. Ioo de signification.

I a simple répétition des différences dans le même sens pendant quatre années (pour l'âge au premier œuf et la ponte jusque fin novembre) surl'héritabilité moyenne $\left(\frac{h^{2}{ }_{d}+h^{2}}{2}\right)$ est de toute façon suggestive (probabilité I/I 6 , pour chacun des caractères, dans l'hypothèse où le hasard seul est cause des différences) ${ }^{1}$ ).

Ainsi, contrairement à certaines opinions émises, quoique l'éclairement supplémentaire diminue la variabilité phénotypique de la ponte, il semble bieì rendre plus nette la ressemblance entre animaux parents, d’origine génétique.

Quelques études antérieures ont révélé des changements de l'héritabilité quand certaines conditions d'élevage sont modifiées (Mc BRIDE, I958). Elles sont très peu nombreuses, et on ne peut songer à dégager de leur confrontation une vue d'ensemble.

2. - Il se pourrait donc que la sélection donne avec un troupeau " éclairé " des résultats plus rapides que sur un troupeau soumis aux conditions naturelles. Ainsi, dans notre cas, les variances phénotypiques et héritabilités moyennes $\left(\frac{h^{2}{ }_{d}+h^{2}}{2}\right)$ estimées sur les animaux éclairés et non éclairés pour la ponte au $3 \mathrm{I}$ décembre sont respectivement :

$$
\begin{array}{ll}
\sigma_{1_{1}}^{2}=522 & h_{1}^{2}=0,53 \\
\sigma_{1_{2}^{2}}^{2}=540 & h_{2}^{2}=0,3 \mathrm{I}
\end{array}
$$

Mc BRIDE, I958, mentionne que le rapport des gains obtenus en une génération de sélection de même intensité, dans les milieux I et 2 , est donné par

$$
\frac{\Delta \mathrm{G}_{1}}{\Delta \mathrm{G}_{2}}=\frac{\sigma \mathrm{P}_{1}}{\sigma \mathrm{P}_{2}} \cdot \frac{h_{1}^{2}}{h_{2}^{2}}
$$

Si, dans le cas présent, nous nous contentons de l'indication fournie par l'héritabilité moyenne, nous obtenons pour rapport des gains dans les deux milieux :

$$
\frac{\sqrt{522} \times 0,53}{\sqrt{540} \times 0,3 \mathrm{I}}=\mathrm{r}, 68 .
$$

(I) A noter que les estimations des 4 années sont à peu près équivalentes quant à leur précision. 
Le gain prévu de cette manière serait donc de 68 p. Ioo plus grand si les poulettes à sélectionner étaient "éclairées".

Naturellement, ceci n'est qu'un ordre de grandeur assez grossier, vu l'erreur d'échantillonnage de nos estimations. En tous cas, il serait intéressant de vérifier cette tendance par une expérience de sélection comparée.

3. - On peut interpréter ces résultats comme une réduction, par l'éclairement supplémentaire des pondeuses, de la variance dûe à des facteurs non contrôlés du milieu, la sensibilité des individus à ces facteurs étant peut-être en outre génétiquement " non-additive ». Cette interprétation apparaît plausible étant donné ce qu'on sait de la sensibilité plus grande de la ponte à des manipulations, changements de locaux et accidents divers en périodes de jours décroissants, fait d'observation courante.

Ces résultats suggèrent d'autre part - vu le rôle attribué au croisement dans la réalisation de 1" " homéostasie physiologique " (LERNER, I954) - de vérifier si la supériorité des croisements pour la ponte n'est pas moindre chez des animaux éclairés.

Le fait de savoir si une ponte plus intensive réalisée par un éclairement approprié, permettrait une sélection plus rapide, et si, par contre, les conditions naturelles seraient meilleures pour juger de l'effet d'hétérosis des croisements, est d'un intérêt certain pour la pratique de la sélection.

4. - Si l'on regarde le détail des composantes "père " et "mère " de la variance, une certaine tendance semble exister, pour la composante " mère ", à être proportionnellement plus grande en milieu "éclairé". Si cela se confirmait, cela pourrait se rapprocher d'un résultat antérieur sur l'âge au premier œuf (MERAT, I956) qui suggérait une part plus grande des gènes liés au sexe dans la variance totale, chez les lots éclos tard et entrant en ponte dans les jours rapidement décroissants de l'automne.

\section{SUMMARY}

This study was undertaken to verify whether the heritability of several egg-laying traits on pullets is, or not, modified by giving the birds an additional lighting to stimulate their production.

The flock under study, originating from a cross (R. I. R. $\times$ Gâtinaise) in I954, is selected for a number of economic traits since that time, and was rendered homogeneous for several morphological and colour characters.

On 4 successive years (I956, I957, I958, I959), the pullets, hatched in march-april, were housed, one half in an egg-laying house with artificial light added to natural day-light, so as to provide since 20 th August a constant $\mathrm{r}_{4}$ hour " day " (" lighted" pullets); the other half was placed in an identical house, but whith only natural daylight. (" not lighted " pullets).

Pullets hatched the same day were, at ro weeks of age, attributed to one 
or the other laying house according to whether their wing-number was odd or even. Each family was, by this way, approximately divided in two halves, each in one house.

The laying house chosen to be artificially lighted was alternated from one year to the following (cf. page 3 : "Material ").

There are approximately, on the whole, I 200 pullets in each group (lighted or not).

The traits studied are : Age at 1st egg, and number of eggs laid respectively till the end of october, november and december of the same year, by the survivors.

The variance components and heritabilities were estimated according to the model described in 1954 by KING and HENDERSON, for the case of unequal numbers in the subclasses. The " hatch " source of variation was taken into account in I957, I958 and I959, by separating the hatching period (maximum 6 weeks) in 2 halves. This effect is, also, removed in the estimation of phenotypic variances.

The results are given in tables I to IV :

Table I : Means and variances for " lighted " and " not lighted " pullets, for the 4 years and 4 traits studied, and pooled data for each trait (with intrayear pooled variances).

Table II : Coefficients of variation.

Table III : Variance components and heritabilities for the 4 years.

Table IV : Variance components and heritabilities, pooled data (on an intra-year basis).

These results show a less phenotypic variance for the "lighted " pullets, especially for age at first egg. The coefficients of variation of that group are also noticeably inferior.

For the heritabilities, they are, in the whole, substantially greater in the " lighted " group, for age at first egg as well as for the numbers of eggs laid : This fact is observed in the 4 years, except for eggs laid till the end of october and december $195^{8}$, where the heritability is approximately equal in the two groups.

On the pooled data, for the 3 I december number of eggs, the mean heritability $\left(\frac{h^{2} d+h^{2}}{2}\right)$ is 0,53 for the "lighted" and 0,3 I for the "not lighted " pullets The corresponding values for age at first egg are 0,44 and 0,17 .

Notwithstanding the absence of an entirely satisfactory test of significance in the case of unequal numbers in the subclasses, this difference in the heritabilities seems to be significant (page 8).

It is suggested, accordingly, that selection for egg-laying, on a lighted flock, could give more rapid results than with only natural daylight. Our data suggest roughly a gain per generation $68 \mathrm{p}$. Ioo higher in the first case. That should be verified by a selection experiment.

\section{RÉFÉRENCES BIBLIOGRAPHIQUES}

Abllanaly H., I953. The relative importance of environment in the evaluation of large families. Poult. Sci., 32, 885 .

Asmundson V. S., Lorentz F. W. et Moses B. D., I946. Influence of light intensity on ovulation in turkeys. Poult.. Sci., 25, 346-354. 
Asmundson V. S. et Moses B. D., I950. Influence of length of day on reproduction in turkey hens. Poult. Sci., 29, 34-4I.

Bissonnette T. H. et CsEch A. G., I938. Interrupted night lighting with pheasants. Ecology, 19, I8I-r87.

Bowman J. C. et ARchibald J. O. H., i959. Effect of controlled lighting on production characters in the fowl. Nature, 183, 2, II38-II39.

Byerty T. C., I948. Light and egg production. Poult. Sci., 27, 656.

Davis G. T. et Penguite R., I942. Sexual activity in Guineas subjected to all-night lights. Poult. Sci., 21, 468-469.

Dobie J. B., Carver J. S. et Rorerts J., I946. Poultry lighting for egg production. Washington Agr. Exp. Sta. Bul., 4 ry.

FAiconer D. S., I952. The problem of environment and selection. Amer. Nat., 86, 293-298.

HARPER J. A., I949. The rate of response of turkey hens to artificial light as related to reproduction. Poult. Sci., 28, 3I2-3I4.

HOFFMANN F. et HANCOCK B., I950. The effect of length of day on egg production of geese and guineas. Poult. Sci., 29, 469-47I.

Hu'Tr F. B., I949. Genetics of the fowl. I ${ }^{\text {re }}$ éd., 590 pp., Mc Graw Hill Book Co, New-York.

KaRAíETJAN S. K., I953. Cité dans Animal Breeding Abstracts, 22, I53 : "L'effet du régime lumineux sur les organes reproducteurs et la production des volailles domestiques ". Agrobiologija, 3, 47-54.

KarapetJan S. K., I954. "Influence de l'augmentation de la durée d'exposition à la lumière sur la prolongation de la vie et de la production des volailles ». Dokl. Akad. Nauk. SSSR., 94, 585-588.

KING S. C. et HENDERSON C. R., I954. Variance components analysis in heritability studies. Poult. Sci., 33, I47-I54.

LFRNER I. M., I954. Genetic homeostasis, Ire éd., I34 pp. Oliver etBoyd, Edinburgh.

MC Bride G., I958. The environment and animal breeding problems. Anim. Breed. Abstr., 26, 4, 349-358.

MÉRAT Ph., I955. Ia race Gâtinaise, étude de sa variabilité génétique en vue d'obtenir des croisements homogènes. Ann. Zoot., 4, 259-283.

Méra'T Ph., I956. L'effet de la date d'éclosion sur le résultat de la sélection pour la précocité sexuelle des volailles. Ann. Zoot., 3, I73-I94.

Nicholas J. E., Cariennbach E. W. et Murphy R. R., I944. I.ight intensity as a factor in the artificial illumination of pullets. Pensylvania $A g r . E x p$. Sta. Bul., 462.

Osborne R., I952. Sexual maturity in Brown I,eghorn. The interaction of genotype and environment. Proc. Roy. Soc. Edinb. B, 54, 445-455.

OSBORNE R., I954. Sexual maturity in Brown Leghorns, and the relationship of genetic variance to differences in environment. Proc. Roy. Soc. Edinb. B., 65, $285-298$.

Osborne R. et PAterson W. S. B., I952. On the sampling variance of heritability estimates derived from variance analyses. Proc. Roy. Soc. Edinb. B., 44, 4, 456-46I.

Romanoff A. I. et Romanofr A. J., I949. The avian egg. J. Wiley et sons, inc. New-York. Chapman et Hall Ltd London.

ScoTT H. M. et PAYNE L. F., I937. Light in relation to the experimental modification of the breeding season of turkeys. Poult. Sci., 16, 90-96.

SKAILIFR F. et SHELDON B. I., I954. Interactions of genotype and environment on determining sexual maturity in the domestic fowl. Austr. Jour. Agric. Res., 6, I7, I7I-I85.

WHETHAM E. O., I933. Factors modifying egg production with special reference to seasonal changes. J. Agr. Sci., 23, 383-4Ix. 
WIICKE H. L., I939. The use of artificial lights for turkeys. Poult. Sci., 18, $236-243$.

Wilson W. O. et Abplanalp H., 1956. Intermittent light stimuli in egg production of chickens. Poult. Sci., 35, 532-538.

WIIson W. O. et WoOdard A. E., I958. Egg production of chickens kept in darkness. Poult. Sci., 3\%, I054-I057. 